\title{
RECTIFIABILITY AND INTEGRALGEOMETRIC MEASURES IN HOMOGENEOUS SPACES ${ }^{1}$
}

\author{
BY JOHN E. BROTHERS
}

\author{
Communicated by Herbert Federer, October 16, 1968
}

1. Introduction. In recent years much of the progress which has been made in geometric measure theory has depended on knowledge of the geometric structure of subsets of $n$ dimensional Euclidean space $R^{n}$ relative to some measure such as the $k$ dimensional Hausdorff measure $H^{k}, 0<k<n$. For example, the proof in [5] of the existence of solutions for the least area problem (Plateau's problem) and the minimal surface problem depends essentially on this structure theory.

Central to the structure theory is the characterization of rectifiable subsets in terms of their projection properties. Such results were obtained first by Besicovitch in [1] for $H^{1}$ in $R^{2}$, then by Federer in [4] for general measures on $R^{n}$. In the present paper we give global generalizations of these theorems to measures on a separable manifold $X$ with a transitive group of diffeomorphisms $G$. The proofs will appear in [3].

Turning to integralgeometric (Favard) measure, we recall that it is easy to determine the relationship between $H^{k}$ and the classical integralgeometric measure on $R^{n}$ once the projection properties of sets of finite $H^{k}$ measure are known and Crofton's formula is verified for $k$ rectifiable sets. We define generalized integralgeometric measures in a manifold of constant curvature and apply our structure theory, together with the integralgeometric formulas of [2], to determine the relationship between $H^{k}$ and these measures.

2. Preliminaries. Recall that in a separable Riemannian manifold the statements " $H^{k}(A)=0$ " and " $A$ is $H^{k}$ sigma finite" are independent of the metric; hence are meaningful for $A \subset X$.

Let $\operatorname{dim} X=n$.

Denote $\alpha(n)=H^{n}\left(R^{n} \cap\{x:|x|<1\}\right)$ and

$$
\beta(n, k)=\left(\begin{array}{l}
n \\
k
\end{array}\right)^{-1} \alpha(n)^{-1} \alpha(k) \alpha(n-k) .
$$

Let $\phi$ be a nonnegative measure on $X$ such that closed sets are $\phi$ measurable.

\footnotetext{
${ }^{1}$ Research partially supported by National Science Foundation grant GP7505.
} 
$R \subset X$ is $k$ rectifiable if there exists a Lipschitzian function mapping some bounded subset of $R^{k}$ onto $R$.

$R \subset X$ is countably $k$ rectifiable if $R$ is the union of a countable family of $k$ rectifiable sets.

$E \subset X$ is countably $(\phi, k)$ rectifiable if there exists a countably $k$ rectifiable set $R$ with $\phi(E \sim R)=0$.

$E \subset X$ is $(\phi, k)$ rectifiable if $E$ is countably $(\phi, k)$ rectifiable and $\phi(E)<\infty$.

$E \subset X$ is purely $(\phi, k)$ unrectifiable if $E$ contains no $k$ rectifiable set $R$ with $\phi(R)>0$.

Let $B$ be a proper $n-k$ dimensional submanifold of class 1 of $X$.

3. The structure theorems. Suppose one of the following two conditions is satisfied.

(i) $X$ is a Riemannian manifold with isometry group $G$; $\operatorname{dim} G$ $=\frac{1}{2} n(n+1)$.

(ii) $X$ has a $G$ invariant linear connection, $B$ is totally geodesic, and for $o \in X$

$$
G \cap\{g: g(0)=0\}
$$

acts transitively on the $n-k$ dimensional linear subspaces of the tangent space of $X$ at $o$.

Let $\Psi$ be a Haar measure on $G$.

THEOREM 1. If $E \subset X$ is $H^{k}$ sigma finite, then there exists a countably $k$ rectifiable Borel subset $R$ of $X$ such that $E \sim R$ is purely $\left(H^{k}, k\right)$ unrectifiable and

$$
(E \sim R) \cap g(B)=\varnothing
$$

for $\Psi$ almost all $g \in G$. Furthermore, if $H^{k}(E \cap R)>0$ and $E$ is $H^{k}$ measurable, then

$$
H=G \cap\{g: E \cap R \cap g(B) \neq \varnothing\}
$$

is $\Psi$ measurable and $\Psi(H)>0$.

If $W \subset X$ and $x \in X$, then

$$
\Theta^{* k}(\phi, W, x)=\limsup _{r \rightarrow 0^{+}} \alpha(k)^{-1} r^{-k} \phi(W \cap\{y: \operatorname{dist}(x, y)<r\})
$$

is the $k$ dimensional upper $\phi$ density of $W$ at $x$.

Corollary. Suppose $W \subset X, \phi(W)<\infty, \phi(S)=0$ whenever $S \subset W$ 
and $H^{k}(S)=0$, and

$$
\Theta^{* k}(\phi, W, x)>0 \quad \text { for } \phi \text { almost all } x \in W \text {. }
$$

Then there exists a countably $(\phi, k)$ rectifiable and $\phi$ measurable set $Q$ such that $W \sim Q$ is purely $(\phi, k)$ unrectifiable and

$$
(W \sim Q) \cap g(B)=\varnothing
$$

for $\Psi$ almost all $g \in G$. Furthermore, if $\phi(W \cap Q)>0$ and $W$ is a Borel set, then there exists a $\Psi$ measurable subset $H$ of $G$ such that $\Psi(H)>0$ and

$$
W \cap Q \cap g(B) \neq \varnothing
$$

whenever $g \in H$.

4. Integralgeometric measures. Assume that condition (i) of $\$ 3$ holds. Choose $o \in X$ and define $\pi: G \rightarrow X$ by $\pi(g)=g(0)$. There is a Haar measure $\Psi$ on $G$ such that for $S \subset X$,

$$
\Psi\left[\pi^{-1}(S)\right]=H^{n}(S) \text {. }
$$

Since $G$ is unimodular, $\Psi$ is independent of choice of 0 .

Theorem 2. Assume $H^{n-k}(B)<\infty$. There exists a Borel regular measure $g_{B}$ on $X$, the integralgeometric measure corresponding to $B$, such that if $A$ is a Borel subset of $X$, then

$$
\mathscr{g}_{B}(A)=\beta(n, k)^{-1} H^{n-k}(B)^{-1} \int_{G} H^{0}[A \cap g(B)] d \Psi g .
$$

Furthermore, $\Phi_{B}(W)=0$ whenever $H^{k}(W)=0$.

Theorem 3. Suppose $H^{n-k}(B)<\infty, A \subset X$ and $H^{k}(A)<\infty$.

(i) If $A$ is purely $\left(H^{k}, k\right)$ unrectifiable, then $\mathscr{I}_{B}(A)=0$.

(ii) $H^{k}(A) \geqq g_{B}(A)$, with equality if and only if $A$ is $\left(H^{k}, k\right)$ rectifiable.

This is an immediate consequence of Theorems 1 and 2 and $[2,5.15]$.

Let $\varepsilon$ be a set of closed $n-k$ dimensional submanifolds of $X$ such that $G$ actstransitively on $\mathcal{E}$ and such that if $E \in \mathcal{E}$, then $G \cap\{g: g(E)=E\}$ is transitive on $E$. Also assume that $\mathcal{E}$ has a $G$ invariant Haar measure.

If $X$ is connected, then $X$ is isometric to either an Euclidean space, a sphere, a real projective space or a simply connected hyperbolic space. For each of these spaces $\mathcal{E}$ can be taken to be the set of totally geodesic submanifolds of dimension $n-k$. 
TheOREM 4. There exists a Haar measure $\Phi$ on $\&$ and a Borel regular measure $g_{\varepsilon}$ on $X$, the integralgeometric measure corresponding to \&, such that if $A$ is a Borel subset of $X$, then

$$
g_{\varepsilon}(A)=\beta(n, k)^{-1} \int_{\varepsilon} H^{0}(A \cap E) d \Phi E .
$$

If $E \in \mathcal{E}$, then there is an open subset $\beta$ of $E$ such that $g_{8}=g_{\beta}$.

If $X=R^{n}$ and $\varepsilon$ is the set of $n-k$ planes, then $g_{g}$ is the $k$ dimensional integralgeometric measure defined in [4].

Theorem 5. Assume $A \subset X$ with $H^{k}(A)<\infty$.

(i) If $A$ is purely $\left(H^{k}, k\right)$ unrectifiable, then $g_{\varepsilon}(A)=0$.

(ii) $H^{k}(A) \geqq \mathfrak{g}_{\mathcal{\varepsilon}}(A)$, with equality if and only if $A$ is $\left(H^{k}, k\right)$ rectifiable.

This is an immediate consequence of Theorems 3 and 4.

\section{REFERENCES}

1. A. S. Besicovitch, On the fundamental geometrical properties of linearly measurable plane sets of points. III, Math. Ann. 116 (1939), 349-357.

2. J. E. Brothers, Integral geometry in homogeneous spaces, Trans. Amer. Math. Soc. 124 (1966), 480-517.

3. - The $(\phi, k)$ rectifiable subsets of a homogeneous space, Acta Math. (to appear).

4. H. Federer, The $(\phi, k)$ rectifiable subsets of $n$ space, Trans. Amer. Math. Soc. 62 (1947), 114-192.

5. H. Federer and W. H. Fleming, Normal and integral currents, Ann. of Math. (2) 72 (1960), 458-520.

INDIANA UNIVERSITY, BLOOMINGTON, INDIANA 47405 